\title{
CONFIABILIDAD DE LOS TEST QUE MIDEN LAS CAPACIDADES COORDINATIVAS EN DEPORTES ACÍCLICOS.
}

STRENGTH TRAINING WITH THE VASCULAR OCLUSION METHOD IN STUDENTS OF SPORTS SCIENCES.

\section{Felipe Cardona Triana}

Profesional en Ciencias del Deporte

Universidad de Ciencias Aplicadas y Ambientales

U.D.C.A

E-mail: pipecartri7@gmail.com

\section{Jorge Enrique Buitrago Espitia}

Doctor en Ciencias de la Cultura Física

Docente Universidad de Ciencias Aplicadas y

Ambientales U.D.C.A

E-mail: jorge.buitrago@udca.edu.co

\section{RESUMEN}

Objetivo: Determinar la confiabilidad de las pruebas empleadas para medir las capacidades coordinativas. Metodología: Se realizó la búsqueda de un total de 35 artículos mediante una revisión bibliográfica en bases de datos como: Sport Discus, Ebsco, Proquest, Science Direct y Google Académico. Efectuada la búsqueda de los artículos, se realizó una tabla en Excel 2013 para organizarla en forma de base de datos. Se seleccionaron 30 de los 35 artículos teóricos y empíricos acerca de los test empleados para medir las capacidades coordinativas en deportes acíclicos y actividades físicas donde se analizaron y se determinaron los test más utilizados para medir las capacidades coordinativas. Resultados: se evaluaron todos los artículos en términos de protocolo, fiabilidad, validez y objetividad con el fin de valorar la confiabilidad de las pruebas en una escala de 0 a 3. A partir de la valoración efectuada se evidencia que el Star excursion balance test (SEBT), Y Balance Test (YBT) se perfilan como las más adecuadas para medir el equilibrio. En cuanto a la capacidad de reacción el test de Galton es un test de fácil aplicación, sin embargo los parámetros de confiabilidad no son los más adecuados para medir la reacción. El Illinois ball Dribbling Test, Coda Test y Arrowhead Agility Test sobresalen como las más adecuadas para medir las capacidades coordinativas en deportes específicos. Mientras que el MABC-2 Test, y KTK Test se destacan como las más adecuadas para medir varias capacidades coordinativas. Finalmente, el T-Test, Illinois Test y 505 Agility Test se perfilan como las más adecuadas para medir la agilidad.

Palabras Claves: Capacidades coordinativas, test motor, deporte de equipo, adquisición de habilidades, desarrollo del deportista.

\section{ABSTRACT}

Aim: To determine the reliability of the tests used to measure the coordination abilities. Method: A total of 35 articles were searched through a bibliographic review in 
databases such as: Sport Discus, Ebsco, Proquest, Science Direct and Google Scholar. After the search of the articles, a table was made in Excel 2013 in order to organize it in the form of a database. Thirty of the 35 theoretical and empirical articles were selected about the tests used to measure the coordination abilities in acyclic sports and physical activities. After that, the articles were analyzed and it was determined which one of the tests was the most used to measure the coordination abilities. Results: The articles were evaluated in terms of protocol, reliability, validity and objectivity in order to assess the reliability of the tests on a scale of 0 to 3.From the assessment made it is evident that the Star excursion balance test (SEBT), and $Y$ Balance Test (YBT) are outlined as the most appropriate to measure the balance. Regarding the reaction ability, the Galton test is an easy application test, however the reliability parameters are not the most adequate to measure the reaction. The Illinois Ball Dribbling Test, Coda Test and Arrowhead Agility Test stand out as the most appropriate to measure the coordination skills in specific sports. While, the MABC-2 Test, and KTK Test stand out as the most suitable to measure various coordination abilities. Finally, the TTest, Illinois Test and 505 Agility Test are outlined as the most appropriate to measure agility.

Key words: Coordination abilities, motor test, team sports, skill acquisition, player development.

\section{INTRODUCCIÓN}

El desarrollo de las capacidades coordinativas es importante para la práctica deportiva porque contribuye al mejor desempeño del deportista, una dificultad que enfrentan los profesores y entrenadores deportivos es medir con objetividad el desarrollo de las capacidades coordinativas; por tal motivo, es importante contar con test que tengan validez, fiabilidad y objetividad.

De acuerdo con Weineck (2005), las capacidades coordinativas permiten organizar y regular el movimiento y se interrelacionan con las habilidades motrices, tanto básicas como deportivas, y sólo se hacen efectivas en el rendimiento deportivo por medio de su unidad con las capacidades físicas. Por tal razón, es esencial desarrollar las capacidades coordinativas a una edad apropiada (Adalberto, 2006).

En los deportes acíclicos, los entrenadores, y preparadores físicos encargados de la preparación deportiva en la etapa formativa y de especialización, se centraron en el desarrollo y perfeccionamiento de las capacidades condicionales tales como: la fuerza, la resistencia y la velocidad, dejando de lado las capacidades coordinativas (Camacho, 2004).

Con el paso del tiempo y la evolución que ha tenido el deporte en todos los niveles, los profesionales en ciencias del deporte ampliaron su horizonte hacia otras capacidades íntimamente relacionadas con el sistema nervioso central. Estas son las capacidades coordinativas, que permiten al deportista realizar movimientos con precisión, economía y eficacia (Verkhoshansky, 2001). 
El grado de coordinación del ser humano, está determinado entonces, por el desarrollo del sistema nervioso central y por las experiencias motrices que haya tenido a lo largo de su desarrollo físico, en especial en el período comprendido entre los siete y 13 años, debido a que el desarrollo de estas capacidades en esta etapa permite el desarrollo de la coordinación y también, el de otras cualidades físicas como la fuerza, velocidad, resistencia, flexibilidad, entre otros. De ahí, que el desarrollo adecuado de las capacidades coordinativas a edades tempranas, posibilita en los niños el perfeccionamiento de sus capacidades de adaptarse de forma motriz a una determinada situación e influyen de distinta manera en el desarrollo general del deportista (Martin, 2001).

Debido a esto, el entrenamiento de las capacidades coordinativas especialmente en edades tempranas es de vital importancia porque como lo menciona Jacob (2017), es un elemento que condiciona la vida en general, el aprendizaje motor y el alto rendimiento deportivo, adicionalmente su correcta evaluación y seguimiento serán fundamentales para el desarrollo motor y competitivo de los deportistas emergentes.

Por ende, es importante efectuar el análisis y la revisión en la metodología de los test existentes para así valorar los protocolos y determinar si cumplen con los mínimos criterios de calidad, de manera que los resultados obtenidos de la aplicación del test en particular, sea una medida fiable.

La necesidad de identificar los criterios de fiabilidad, validez y objetividad de los test empleados para medir las capacidades coordinativas y los escasos estudios que valoren los test existentes, constituyen la contradicción que deriva la situación problemática de este trabajo.

Se plantea entonces, el siguiente problema de investigación ¿Qué confiabilidad tienen las pruebas empleadas para medir las capacidades coordinativas?

Mediante este trabajo se evalúan los test existentes para medir las capacidades coordinativas en deportes acíclicos. A partir de la valoración de la calidad de las pruebas analizadas, esta investigación provee a los profesionales en áreas afines al deporte, información valiosa para seleccionar test confiables que permitan controlar el desarrollo de estas capacidades. Se contribuye además, con la recolección de los postulados de los autores más importantes existentes sobre cómo desarrollar y controlar las capacidades coordinativas.

Se utilizaron cinco bases de datos: 1.Sport Discus, 2. Ebsco, 3.Proquest, 4. Science Direct y 5. Google Académico con el propósito de acceder a los artículos publicados. Para realizar la búsqueda en estas bases de datos se utilizaron los siguientes descriptores: «coordination abilities», «motor test», «team sports», «skill acquisition», y «player development»

Con el propósito de centrar la búsqueda en el objeto de estudio, se emplearon criterios de inclusión: Artículos que aborden niños, jóvenes y adultos entre los 6 y 30 años de edad; experiencia deportiva de los sujetos de mínima de un año; estuidos de deportes acílicos en equipo (Fútbol, baloncesto, voleibol y balónmano); publicaciones entre los años 2006 y 2018 en español e inglés. 


\begin{tabular}{lllll}
\hline $\begin{array}{l}\text { En la siguiente tabla se relaciona la búsqueda realizada de } \\
\text { consultados por base de datos.Tabla 1. Revisión de artículos }\end{array}$ & artículos \\
$\begin{array}{l}\text { Base de datos } \\
\text { Encontrados }\end{array}$ & Idioma & Descargados & $\begin{array}{l}\text { Seleccionado } \\
\text { s }\end{array}$ \\
\hline Sport Discus & 12 & 12 Inglés & 12 & 10 \\
\hline Ebsco & 6 & 6 Inglés & 6 & 6 \\
\hline Proquest & 3 & 3 Inglés & 3 & 2 \\
\hline Science Direct & 8 & $\begin{array}{l}\text { 7 Inglés } \\
\text { 1 Español }\end{array}$ & 8 & 7 \\
\hline Google Académico & 6 & $\begin{array}{l}\text { 5 Inglés } \\
\text { 1 Español }\end{array}$ & 6 & 5 \\
\hline Total artículos & 35 & $\begin{array}{l}33 \text { Inglés } \\
\text { 2 Español }\end{array}$ & 35 & 30 \\
\hline
\end{tabular}

(Artículos revisados. Elaboración propia)

De los 35 artículos encontrados, se analizan 30 artículos teóricos y empíricos acerca de los test empleados para medir las capacidades coordinativas en deportes acíclicos y actividades físicas.

\section{METODOLOGÍA}

De acuerdo Martínez, Zagalaz y Linares (2003), los criterios de calidad orientan el grado de eficiencia de una prueba; su componente cuantitativo se enuncia a través de los tres principales indicadores: Capacidad de objetividad, capacidad de fiabilidad y capacidad de validez. Un aspecto general de gran relevancia es garantizar que entre las administraciones ejecutadas por un mismo examinado o por varios examinandos no haya ningún efecto de entrenamiento por parte de los mismos, ya que esto podría restar fiabilidad y objetividad a la aplicación de la prueba.
El término objetividad se define según Martínez (2002), como "cualidad que lleva a emitir un juicio sin dejar que intervengan preferencias personales; ausencia de prejuicios; imparcialidad".

Un test posee más objetividad en cuanto mayor sea el grado de independencia sobre elementos externos que puedan intervenir. Además, la objetividad debe analizarse de forma aislada, porque se puede perturbar de forma diferente a cada fase de una prueba; en la ejecución de la misma, en su evaluación o en su interpretación. Una prueba objetiva tiene que garantizar que su ejecución se realice con un método y que éste pueda reproducirse consecutivamente de la misma manera. Se puede hablar de una prueba con mayor objetividad, cuanto más medible, en términos numéricos y de acuerdo a escalas estandarizadas, sea su resultado (Martínez, 2002). 
En psicometría según Martínez (2002), la fiabilidad de un test se valora por la coherencia de los resultados conseguidos en dos aplicaciones de la misma prueba o mediante la aplicación de dos formas equivalentes de la prueba a los mismos examinada.

Se deben examinar tres aspectos importantes en el concepto de fiabilidad:

1. Calidad del material utilizado

2. Las técnicas y metodología empleada

3. Tiempo de fiabilidad de la prueba

La validez según Martínez (2002), se define como "la cualidad de un test o prueba que mide realmente lo que se propone medir"

Para Bosco como se citó en Martínez, (2002), "el éxito y el valor de un test depende en gran manera de la estandarización, de tal modo que debe dar la posibilidad de ser utilizado por cualquier persona (lógicamente debe ser un experto), y en cualquier situación".

El procedimiento seguido para la organización y análisis de la información siguió tres pasos, en el primero se reúne la información para facilitar el acceso, consulta y eliminar la información repetida; en el segundo paso se elabora una tabla de análisis que incluye las categorías para valorar los aspectos más relevantes de cada artículo; por último, se clasifica cada artículo por la capacidad coordinativa que mide.

\section{Indicadores metodológicos de los test} referidos en los artículos consultados.

De los 30 artículos consultados se obtienen 20 test diferentes empleados para medir las capacidades coordinativas en actividades físicas y deportes acíclicos, los cuales se agrupan para su análisis de acuerdo con la capacidad que miden: para la capacidad de equilibrio se encontraron tres test, uno que mide la capacidad de reacción, seis test específicos en deportes acíclicos, dos test que miden varias capacidades coordinativas, y ocho dirigidos a valorar la agilidad.

\section{Análisis de los test}

Para el análisis de cada test, se utilizó una hoja de cálculo de Excel de Microsoft 2013. Para ello se concedió a cada uno de los test una puntuación en función del grado de cumplimiento sobre cada uno de los criterios de protocolo de la prueba, fiabilidad, objetividad y validez. De este modo, los datos se pudieron manejar y tratar estadísticamente.

En la tabla siguiente se exponen los criterios propuestos por Sanchez-Pay, (2011), a partir de los cuales se realizó una evaluación de cada test. 
Tabla 2. Descripción de los criterios de confiabilidad que debe cumplir un test físico. Criterio Descripción

Manera de proceder por parte del evaluador a la hora de realizar un test.

Protocolo Para ello, será necesario que exista: a) una descripción de la prueba, de la

de

actuación c) del material (si lo hubiese) y la forma de utilización; y d) la forma de medición, puntuación, y penalización si las hubiese.

Grado de independencia que posee una prueba, sobre elementos externos que puedan intervenir en ella. Se debe garantizar que su ejecución se realice con arreglo a un método, y que éste se pueda reproducir posteriormente de la

Objetividad misma manera (Martínez, 2006). La objetividad se mide a través de un coeficiente. Dicho coeficiente de objetividad resultará de la correlación de los resultados obtenidos por distintos examinadores con los mismos examinados e iguales consignas (Fetz y Kornexl, citados en Martínez, 2006).

Grado de exactitud con el que el test mide una marca característica Fiabilidad determinada, independiente de si el test requiere o no medir esa marca (Haag y Dassel, como se citó en Sanchez-Pay, 2011). Existen diversos factores que afectan a dicho criterio. Para controlar la fiabilidad de una prueba, se debe tener en cuenta: la calidad del material utilizado, las técnicas y la metodología empleada, y el tiempo de fiabilidad de la prueba (Martínez, 2006). Los índices de fiabilidad no se presentan específicamente para cada test.

Grado de precisión con la que el test mide aquello que debe medir. Se suele Validez obtener de la correlación de la marca en un test, y el rendimiento obtenido en una acción técnica que tenga similitud a la forma de ejecución del test. Los índices de validez no se presentan específicamente para cada test.

Fuente: Adaptado de Sanchez-Pay, A., Torres-Luque, G., Palao, J. (2011). Revisión y análisis de los test físicos empleados en tenis. European Journal of Human Movement, 26, 104-122.

\section{RESULTADOS Y DISCUSIÓN}

\section{Resultados}

La valoración se realiza siguiendo el orden en que se agruparon los test consultados, a continuación se exponen los resultados obtenidos: 
Tabla 3. Análisis de los test empleados para medir la capacidad de equilibrio en deportes acíclicos

\begin{tabular}{|c|c|c|c|c|c|}
\hline & Protocolo & Objetividad & Fiabilidad & Validez & Total \\
\hline Test de equilibrio & Escala 0-3 & Escala 0-3 & Escala $0-$ & Escala 0- & $\begin{array}{l}\text { Escala } \\
0-12\end{array}$ \\
\hline $\begin{array}{l}\text { Silgle-Leg-Hop- } \\
\text { StabilitationDtest }\end{array}$ & 3 & 2 & 3 & 2 & 10 \\
\hline $\begin{array}{lr}\text { Star } & \text { excursion } \\
\text { balance } & \text { test } \\
\text { (SEBT) } & \end{array}$ & 3 & 3 & 3 & 3 & 12 \\
\hline $\begin{array}{l}\text { Y Balance Test } \\
\text { (YBT) }\end{array}$ & 3 & 3 & 3 & 3 & 12 \\
\hline
\end{tabular}

Fuente: Elaboración propia.

Respecto a los test consultados empleados para medir la capacidad de equilibrio; en la valoración del protocolo las tres pruebas valoradas obtienen la máxima valoración posible, lo que indica que en estos test se describe correctamente la prueba, la acción que debe realizar el sujeto evaluado en la prueba y el evaluador, se relaciona el material necesario para realizar la prueba y la forma de utilizarlo; así mismo, la forma de medición, puntuación y penalizaciones son fácilmente comprensibles.

Respecto a la objetividad, el test Silgle-LegHop Stabilitation no posee un método claramente definido por lo cual al reproducir la aplicación del test.

Los resultados obtenidos difieren levemente. Por su parte, las pruebas Star excursión balance test (SEBT), Y Balance Test (YBT) cumplen los criterios de objetividad valorados.

En la valoración de la fiabilidad, las tres pruebas analizadas miden con exactitud el equilibrio, la calidad del material que utilizan es la adecuada, la técnica y metodología que emplean es óptima.

La validez de la prueba Silgle-LegHop Stabilitation, en relación directa con la objetividad, no es la mejor, debido esencialmente a la inestabilidad en la ejecución de la prueba. Por su parte las pruebas Star excursión balance test (SEBT), Y. Balance Test (YBT) cumplen los criterios de validez valorados. 
En síntesis, a partir de la valoración efectuada se evidencia que las pruebas Star excursion balance test (SEBT), Y Balance Test (YBT) se perfilan como las más adecuadas para medir el equilibrio.Tabla 4. Análisis de los test empleados para medir la capacidad de reacción en deportes acíclicos.

\begin{tabular}{|c|c|c|c|c|c|}
\hline & Protocolo & Objetividad & Fiabilidad & Validez & Total \\
\hline $\begin{array}{l}\text { Test } \\
\text { reacción }\end{array}$ & Escala 0 a 3 & Escala 0 a 3 & Escala 0 a 3 & Escala 0 a 3 & Escala 0 a 12 \\
\hline Test de Galton & 1 & 2 & 2 & 2 & 7 \\
\hline
\end{tabular}

En los test consultados empleados para medir la capacidad de reacción; la valoración del protocolo la prueba analizada no posee parámetros claros y es por esto que la prueba obtiene una valoración baja, lo que indica que en este test no se describe correctamente la prueba, se describe de forma muy vana la acción que debe realizar el sujeto evaluado en la prueba y el evaluador, no es muy claro cómo se relaciona el material necesario para realizar la prueba y la forma de utilizarlo; además, la forma de medición, puntuación penalizaciones no son fácilmente comprensibles.

Respecto a la objetividad, el test de Galton no posee un método claramente definido por lo cual al reproducir la aplicación del test, los resultados obtenidos difieren levemente. Por su parte. En la valoración de la fiabilidad, la

prueba analizada mide con exactitud la capacidad de reacción de uno de los segmentos corporales más importantes, la calidad del material que utilizan es de nivel medio, la técnica y metodología que emplean es medianamente óptima. La validez de la prueba del Bastón de Galton, en relación directa con la objetividad, no es la mejor, debido esencialmente a la inestabilidad en la ejecución de la prueba. En síntesis, a partir de la valoración efectuada se evidencia que la prueba de Galton es un test sencillo en donde esta capacidad puede evaluarse fácilmente, sin embargo los resultados pueden diferir entre los sujetos evaluados ya que no los parámetros de confiabilidad no son los más adecuados para medir la reacción. 
Tabla 5. Análisis de los test empleados para medir las capacidades coordinativas en deportes acíclicos.

\begin{tabular}{|c|c|c|c|c|c|}
\hline & Protocolo & Objetividad & Fiabilidad & Validez & Total \\
\hline $\begin{array}{l}\text { Test en } \\
\text { específicos }\end{array}$ & Escala 0 a 3 & Escala 0 a 3 & $\begin{array}{l}\text { Escala } 0 \text { a } \\
3\end{array}$ & $\begin{array}{l}\text { Escala } \\
0 \text { a } 3\end{array}$ & $\begin{array}{l}\text { Escala } \\
0 \text { a } 12\end{array}$ \\
\hline Lane Agility Drill Test & 1 & 2 & 1 & 1 & 5 \\
\hline $\begin{array}{l}\text { Test zigzag sprint in half } \\
\text { court }\end{array}$ & 1 & 1 & 1 & 1 & 4 \\
\hline Illinois ball dribbling test & 2 & 2 & 2 & 2 & 8 \\
\hline CODA Test & 2 & 2 & 2 & 2 & 8 \\
\hline AFL Agility Test & 1 & 2 & 1 & 1 & 5 \\
\hline Arrowhead Agility Test & 2 & 2 & 2 & 2 & 8 \\
\hline
\end{tabular}

En relación con los test consultados empleados para medir las capacidades coordinativas en actividades físicas y deportes acíclicos; en la valoración del protocolo tres de las seis pruebas valoradas obtienen una valoración muy baja, lo que indica que en estos test no se describe visiblemente la prueba, la acción que debe realizar el sujeto evaluado en la prueba y el evaluador es poco notoria, el material necesario para realizar la prueba y la forma de utilizarlo no se relacionan completamente; adicionalmente, la forma de medición, puntuación y penalizaciones no son fácilmente comprensibles.

Respecto a la objetividad, el test zigzag sprint in half court no posee un método claramente definido por lo cual al reproducir la aplicación del test, los resultados obtenidos difieren levemente. Por su parte, las pruebas Illinois Ball Dribbling Test, Coda Test y Arrowhead Agility test cumplen con los criterios de objetividad valorados más altos de los prueba valoradas.
En la valoración de la fiabilidad, ninguna de las seis pruebas analizadas obtuvo el máximo puntaje debido a que no es muy claro si los test específicos miden de manera independiente con exactitud las capacidades coordinativas, sin embargo en tres de las seis pruebas la calidad del material que utilizan es la adecuada, la técnica y metodología que emplean es óptima.

La validez de la prueba zigzag sprint in half court, en relación directa con la objetividad, no es la mejor, debido esencialmente a la inestabilidad en la ejecución de la prueba. Por su parte las pruebas Illinois Ball Dribbling test, Coda Test y Arrowhead Agility test son las que mejor cumplen los criterios de validez valorados.

En síntesis, a partir de la valoración efectuada se evidencia que las pruebas Illinois ball Dribbling Test, $C$ oda Test y Arrowhead Agility Test se perfilan como las más adecuadas para medir las capacidades coordinativas en deportes específicos. 
Tabla 6. Análisis de los test empleados para medir más de una capacidad coordinativa en deportes acíclicos.

\begin{tabular}{lccccc}
$\begin{array}{l}\text { Test que miden varias } \\
\text { capacidades }\end{array}$ & Protocolo & Objetividad & Fiabilidad & Validez & Total \\
& Escala 0 a 3 & Escala 0 a 3 & Escala 0 a 3 & Escala 0 a 3 & $\begin{array}{l}\text { Escala 0 a } \\
12\end{array}$ \\
\hline MABC-2 Test & 3 & 3 & 3 & 3 & 12 \\
\hline KTK Test & 3 & 3 & 3 & 3 & 12 \\
\hline
\end{tabular}

Respecto a los test claramente definido cumpliendo los criterios consultados empleados para medir de objetividad valorados. En la valoración de varias capacidades coordinativas; en la la fiabilidad, las dos pruebas analizadas valoración del protocolo las dos pruebas miden con exactitud las distintas capacidades valoradas obtienen la máxima valoración coordinativas, la calidad del material que posible, lo que indica que en estos test se utilizan es la adecuada, la técnica y describe correctamente la prueba, la acción que debe realizar el sujeto evaluado en la prueba y el evaluador, se relaciona el material necesario para realizar la prueba y la forma de utilizarlo; así mismo, la forma de medición, puntuación y penalizaciones son fácilmente comprensibles.

Respecto

a

la objetividad, ambos test poseen un método metodología que emplean es óptima. En cuanto a la validez las pruebas MABC-2 Test, y KTK Test cumplen los criterios de validez valorados.

En síntesis, a partir de la valoración efectuada se evidencia que las pruebas MABC-2 Test, y KTK Test se perfilan como las más adecuadas para medir varias capacidades coordinativas.

Tabla 7. Análisis de los test empleados que miden la capacidad de agilidad en deportes acíclicos.

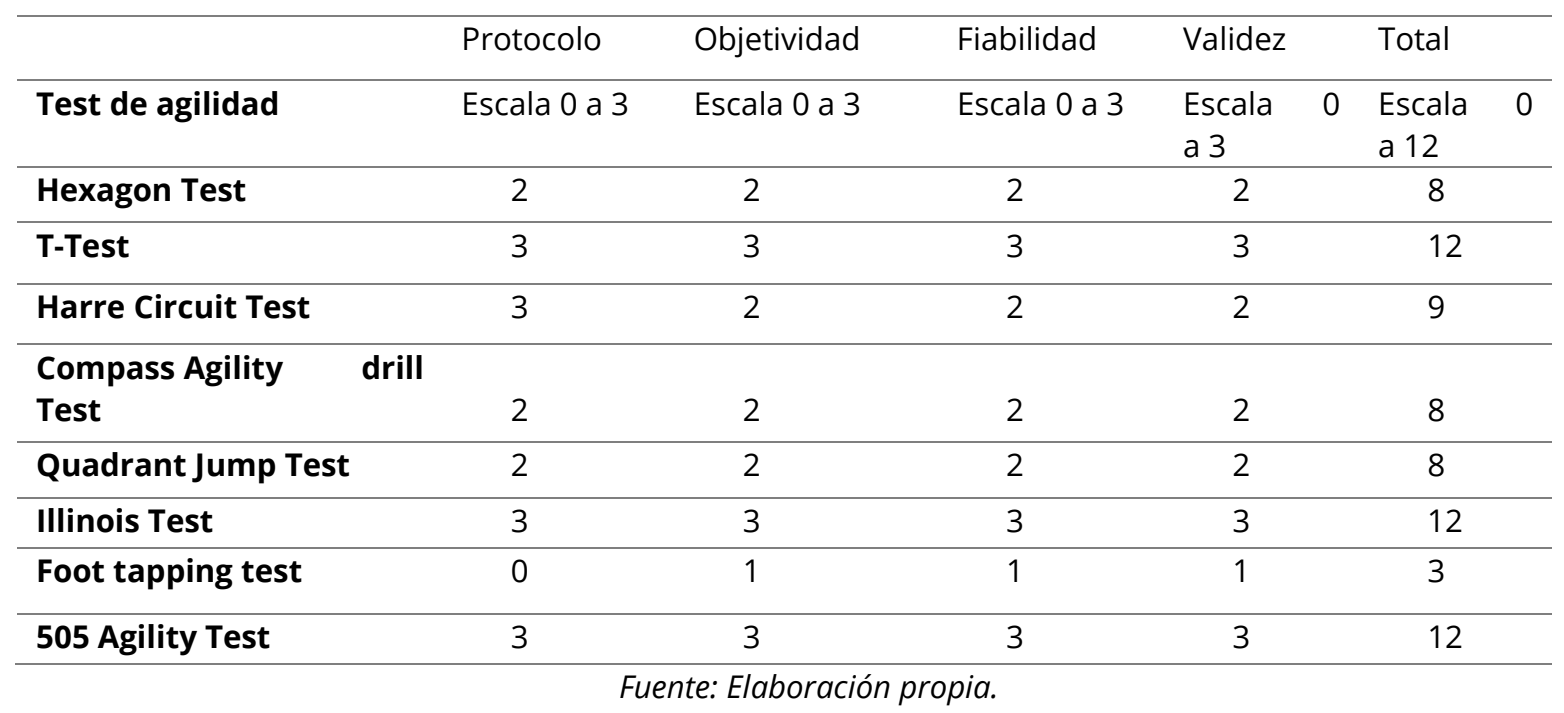


En relación a los test que miden la capacidad de agilidad en actividades físicas y deportes acíclicos; en la valoración del protocolo tres de las ocho pruebas valoradas obtienen la máxima valoración posible, lo que indica que en estos test se describe correctamente la prueba, la acción que debe realizar el sujeto evaluado en la prueba y el evaluador, se relaciona el material necesario para realizar la prueba y la forma de utilizarlo; así mismo, la forma de medición, puntuación y penalizaciones son fácilmente comprensibles.

Respecto a la objetividad, el Foot Tapping Test no posee un método claramente definido por lo cual al reproducir la aplicación del test, los resultados obtenidos difieren levemente. Por su parte, las pruebas TTest, Illinois Test y 505 Agility Test cumplen los criterios de objetividad valorados.

En la valoración de la fiabilidad, las tres pruebas analizadas anteriormente mencionadas miden con exactitud el equilibrio, la calidad del material que utilizan es la adecuada, la técnica y metodología que emplean es óptima.

La validez de la prueba Foot Tapping Test, en relación directa con la objetividad, no es la mejor, debido esencialmente a la inestabilidad en la ejecución de la prueba. Por su parte las pruebas T-Test, Illinois Test y 505 Agility Test cumplen los criterios de validez valorados.

En síntesis, a partir de la valoración efectuada se evidencia que las pruebas TTest, Illinois Test y 505 Agility Test se perfilan como las más adecuadas para medir la agilidad.

\section{DISCUSIÓN}

Martínez, E., Zagalaz, M. y Linares, D. (2003), en su estudio sobre las pruebas de aptitud física en la evaluación de la Educación física de la ESO (Educación Secundaria Obligatoria), refieren la importancia de valorar las capacidades físicas del individuo en tres ámbitos esenciales: el rendimiento deportivo, la educación física escolar, y actividad física para la salud de las personas. Entre las conclusiones del estudio los autores exponen que el 52\% de los 169 profesores encuestados considera que para evaluar la condición física de los adolescentes se deben utilizar PAF (Pruebas de Aptitud Física) que ayuden a valorar las cualidades físicas básicas y motrices del sujeto, el $39 \%$ manifiesta sus dudas sobre la verdadera efectividad de esta práctica y el $9 \%$ se manifiesta en contra. Se destaca que el $94 \%$ de 1.540 alumnos de ESO encuestados contesta afirmativamente a la pregunta anterior, lo cual refuerza aún más la necesidad de utilizar el test pedagógico como elemento de medición y motivador en los estudiantes.

En comparación con los hallazgos de los autores anteriores, se puede afirmar que en la actualidad esta estadística se inclina a favor de la utilización de las pruebas para valorar el nivel de las capacidades físicas, con la necesaria recomendación que su empleo sea individualizado y se eviten las comparaciones con otros sujetos de distintas características, o se califique a partir de valores que no corresponden a los de la población evaluada. 
En su estudio, (Martinez, 2002) valora 167 pruebas (agrupadas por cualidades físicas que miden la resistencia, fuerza, velocidad, flexibilidad, agilidad, coordinación y equilibrio), desde los coeficientes de objetividad, fiabilidad, validez que se obtienen con el análisis de tres frentes simultáneos (aportación de estudios previos ofrecidos por la literatura científica, aportación de los libros de texto, resultados de la encuesta a profesores de Educación Física), cada frente tiene el mismo peso (33.3\%) en la valoración total.

Este estudio constituye referente para este análisis, debido a que las pruebas se valoran a partir de los mismos aspectos de objetividad, fiabilidad, y validez; se señala que en el presente estudio la única fuente empleada ha sido la revisión de la literatura científica y se centra en el análisis de las pruebas para medir las capacidades coordinativas.

En este sentido, Martínez, E., Zagalaz, M. y Linares, D. (2003), evalúan cinco test de coordinación (test de desplazamiento en un zig-zag con balón, test de coordinación dinámica general con cuerda, test de conducción del balón con el pie sobre circuito, test de recepción de objetos móviles, test de slalom con bote de balón). La puntuación puede ser uno o dos puntos. El primer valor se asigna a partir del criterio de los autores que las pruebas responden a la necesidad de medir el estado de forma física y motriz de un individuo, son aplicables a grandes masas, sin necesidad de un material específico; el segundo, si en el test analizado se han obtenido resultados que superen los valores de coeficiente establecidos de objetividad y fiabilidad en análisis individual $=0,85$; coeficiente de objetividad y fiabilidad en análisis grupal $=0,75$; coeficiente de validez mínimo = 0,60.
Los test mencionadas son valorados en los criterios de fiabilidad, objetividad, y validez respectivamente. Al no existir diferencia alguna, es necesario señalar que en la valoración total, la prueba de desplazamiento en un zig-zag con balón alcanza la puntuación de 11,33 situándose en primer lugar, seguida del test de coordinación dinámica general con cuerda 8,44; las tres restantes pruebas obtienen el mismo valor 7,00 cada una.

En las pruebas de equilibrio los autores analizan 15 pruebas (prueba de equilibrio flamenco EUROFIT, prueba de caminar sobre una barra de equilibrio, prueba de equilibrio estático sobre banco, prueba de equilibrio de Komexl, prueba de equilibrio de prueba sentado, test de lowa Brace, prueba de saltos en estrella, prueba de equilibrio de pica de pie, prueba de equilibrio dinámico tras salto giro y caída, test de Bakarinov, test de Dade Country Public School, equilibrio con una sola pierna después de un giro, variante: caminar sobre una pista hexagonal, prueba de equilibrio sobre soporte móvil, prueba de equilibrio de balón sobre puño) (Martinez, 2002).

Los resultados de la valoración ubican en el primer lugar la prueba de equilibrio flamenco EUROFIT con dos puntos en fiabilidad, objetividad y validez respectivamente y un valor total de 14,82; a continuación la prueba de caminar sobre una barra de equilibrio tiene dos puntos en fiabilidad y objetividad, un punto en validez, y total de 12,57; la prueba de equilibrio de Komexl obtiene dos puntos en fiabilidad y objetividad, un punto en validez y total de 9,44; la prueba de equilibrio de prueba sentado se evalúa con dos puntos en fiabilidad y objetividad, un punto en validez y total de 9,19; la prueba de saltos en estrella tiene dos puntos en fiabilidad, un punto en 
objetividad y validez, y total de 8,44; las demás pruebas evaluadas obtienen un punto en cada aspecto, por lo que su posición se determina por el puntaje total.

En otro estudio, Sanchez-Pay, TorresLuque, y Palao (2011), efectúan una revisión y análisis de los test físicos empelados en tenis, a partir de la literatura científica; por lo que es otro referente útil en la comparación con este estudio; los autores valoran 49 test con aplicación al tenis (agrupados en test de resistencia general, test de resistencia específica, test generales de fuerza, test de fuerza específica, test generales de velocidad y agilidad, test específicos de velocidad y agilidad, test de flexibilidad, y test de equilibrio y coordinación).

Los autores valoran el protocolo de la prueba (puntuación de 0 a 4), criterios de calidad (fiabilidad, objetividad, validez; 0 a 12 puntos), valores de referencia normalizados ( 0 a 3 puntos), utilidad y pertinencia ( 0 a 3 puntos), economía y recursos ( 0 a 3 ).

En la valoración de los criterios de calidad los autores dicen respecto a la metodología de evaluación que se distinguen tres criterios de análisis: 1) coeficiente de objetividad: grado de independencia que posee una prueba sobre elementos externos que puedan intervenir en ella; 2) coeficiente de fiabilidad: grado de exactitud con el que el test mide una capacidad y 3) coeficiente de validez: grado de exactitud con la que el test mide aquello que debe medir. Cada criterio fue valorado de 1 a 4 puntos. Para la valoración de los criterios se siguieron las recomendaciones establecidas en la literatura específica (Landis y Koch como se citó en Sanchez-Pay, Torres-Luque, y Palao 2011). De este modo, si el coeficiente se encontraba entre 0 y 0.40 , se valoró como un punto; entre 0.41 y 0.60 con dos puntos; entre 0.61 y 0.80 con tres puntos $y$; entre 0.81 y 1 con cuatro puntos. Esta variable se registró como categórica (Sanchez-Pay, Torres-Luque, y Palao 2011:109)

Centrando el análisis en los test de equilibrio y coordinación, se valora el equilibrio dinámico y coordinación, y el equilibrio estático; ambas pruebas obtienen tres puntos en la evaluación de los criterios de calidad, lo que corresponde a un punto en fiabilidad, objetividad y validez respectivamente. Al observar la puntuación total, la prueba de equilibrio dinámico y coordinación obtiene 13 puntos de 25 posibles, y la prueba de equilibrio estático obtiene 11 puntos. Esta valoración sitúa a ambas pruebas cerca a la media $(12,5)$ de la puntuación máxima posible, por lo que se puede inferir que poseen un valoración discreta que dificulta recomendar con convicción estas pruebas.

Entre las conclusiones del estudio Sanchez-Pay, Torres-Luque, y Palao (2011) dicen que los test generales son los que cumplen un mayor porcentaje de los criterios (coeficientes de objetividad, fiabilidad y validez), que los test específicos de tenis; no obstante ninguno obtiene la puntuación máxima (12 puntos sobre 12). Refieren también, que los protocolos de todos los test analizados cumplen con los criterios necesarios para su aplicación.

A partir de las conclusiones de estos autores, es necesario mencionar que los test generales tienen posibilidad de ser aplicados a poblaciones más amplias y diversas, lo que influye en su rápido perfeccionamiento; en este sentido los test específicos están en desventaja. Sin embargo, en el contexto actual del entrenamiento deportivo, resulta más importante para el entrenador e investigadores obtener información lo más precisa y especializada posible, la cual la aporta los test específicos. 
La información más exacta en la actualidad la aportan los resultados de los test médicos, biomecánicos y fisiológicos específicos, pero sus costos y accesibilidad es muy limitada; por tal motivo, los test pedagógicos específicos se perfilan como una herramienta práctica de gran utilidad y en este sentido su perfeccionamiento bajo los criterios de fiabilidad, objetividad y validez que se establece en la literatura especializada es un aporte importante para el área de las ciencias del deporte.

En el contexto de la valoración de las capacidades coordinativas esta tarea representa un magno esfuerzo, ya que partiendo del hecho en que las capacidades coordinativas están estrechamente relacionadas con las capacidades condicionales y sólo pueden manifestar su desarrollo por medio de ellas, convirtiéndose ambas capacidades realmente en una unidad funcional e inseparable desde el punto de vista práctico (Cañizares, 2001). Es por ello que su evaluación independiente es sumamente complicada afectando el correcto seguimiento y desarrollo motriz del deportista hacia el perfeccionamiento de la técnica deportiva. Según Weineck (2016), estas capacidades permiten organizar y regular el movimiento y se interrelacionan con las habilidades motrices, tanto básicas como deportivas, y sólo se hacen efectivas en el rendimiento deportivo por medio de su unidad con las capacidades físicas.

\section{CONCLUSIONES}

La revisión bibliográfica efectuada confirma el estado de necesidad de valorar la confiablidad de los test que miden las capacidades coordinativas, evidenciada en los escasos estudios realizados; a pesar que los diversos autores consultados coinciden en que las capacidades coordinativas tienen gran importancia para el correcto aprendizaje y el desempeño competitivo.

En su mayoría las pruebas analizadas tienen protocolos bien definidos, sin embargo, son escasas las pruebas que logran medir desde una perspectiva general las capacidades coordinativas, siendo las capacidades que más pruebas disponen para ser valoradas el equilibrio y la reacción; esto evidencia la complejidad que representa medir de manera aislada cada una de las capacidades coordinativas. Se concluyó que los test que tienen mayor confiabilidad para medir la capacidad de equilibrio son el Star excursion balance test (SEBT) y el $\mathrm{Y}$ Balance Test (YBT), en cuanto a la capacidad de reacción según la revisión efectuada los parámetros de confiabilidad del test analizado no son los más adecuados para medir la reacción. Mientras que los test que tienen mayor confiabilidad para medir varias capacidades coordinativas y de agilidad son el MABC-2 Test, KTK Test, el T-Test, Illinois Test y 505 Agility Test respectivamente.

Los criterios utilizados para valorar la confiabilidad de las pruebas que miden las capacidades coordinativas: protocolo de la prueba, coeficientes de fiabilidad, objetividad y validez, referenciados por la literatura especializada son adecuados, por tal motivo, los entrenadores e investigadores deben consultar esta información relacionada con la prueba para obtener información precisa de lo que se quiere medir; también se deben tener en cuenta para la creación de nuevas pruebas o el perfeccionamiento de estas. 


\section{REFERENCIAS BIBLIOGRÁFICAS}

Adalberto, C. (2006). Sistema de capacidades fisicas: Fundamentos teóricos, metodológicos. Rio de Janeiro: Icone.

Alesi, M., Bianco, A., Luppina, G., Palma, A., \& Pepi, A. (2016). Improving children's coordinative skills and executive functions: the effects of a football exercise program. Perceptual and motor skills, 122(1), 27-46.

Alnahdi, A.(2015). Reference values for the $Y$ Balance Test and the lower extremity functional scale in young healthy adults. Journal of Physical Therapy Science, $626-633$.

Benis, R., Bonato, M., \& Torre, A. (2016). Elite Female Basketball Players' Body-Weight Neuromuscular Training and Performance on the Y-Balance Test. Journal of athletic training, 51(9), 688-695.

Bhat, R., \& Moiz, J. (2013). Comparison of dynamic balance in collegiate field hockey and football players using star excursion balance test. Asian journal of sports medicine, 4(3), 221.

Beekhuizen, K., Davis, M., Kolber, M., y Cheng, M. (2009). Test-retest reliability and minimal detectable change of the hexagon agility test. The Journal of Strength \& Conditioning Research, 23(7), 2167-2171.

Camacho. (2004). Pedadogía y didáctica de la educación física. Armenia: Kinesis.

Cañizares. (2016). Coordinación y equilibrio en el niño: Su desarrollo en la edad escolar. Wanceulen.

Henderson, S. (2012). MABC-2, Batería de Evaluación del Movimiento para niños - 2. Madrid, España.

Loan, S. (2016). Testing agility skill at a basketball team (10-12 years old). Science, Movement and Health, Vol. XVI, 103 - 109.

Jacob, W. (2017). El entrenamiento físico en el fútbol como factor de influencia en el aprendizaje del gesto técnico del chut a portería.

Martín, C. (2001). Manual de metodología del entrenamiento deportivo. Paidotribo.

Martínez, E., Zagalaz, L.,Linares, D. (2003). Las pruebas de aptitud física en la evaluación de la Educación física de la ESO. Educación física y deportes, 61-77.

Martínez, J. (2002). Pruebas de aptitud física . Paidotribo.

Plisky, G. (2009). The Reliability of an Instrumented Device for Measuring Components of the Star Excursion Balance Test. North American Journal of Sports Physical Therapy, 92 - 99.

Sánchez-Pay, A. (2011). Revisión y análisis de los test físicos empleados en tenis. European Journal of Human Movement, 105-122.

Torralba, M., Vieira, M., Lleixà, T., y Gorla, J. (2016). Evaluación de la coordinación motora en educación primaria de Barcelona y provincia/Assessment of Motor Coordination in Primary Education of Barcelona and Province. Revista Internacional de Medicina y Ciencias de la Actividad Física y del Deporte, (62). 
Verkhoshansky, Y. (2001). Teoría y metodología del entrenamiento deportivo. Paidotribo. Weineck, J. (2005). Entrenamiento total. Paidotribo.

Weineck, J. (2007). La anatomia deportiva. Paidotribo. 